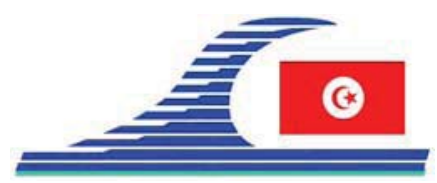

Conférence Méditerranéenne Côtière et Maritime

EDITION 1, HAMMAMET, TUNISIE (2009)

Coastal and Maritime Mediterranean Conference

Disponible en ligne - http://www.paralia.fr-Available online

\title{
Etude et expérimentation de solutions éco-compatibles d'aménagement du littoral à Ravenne, Italie
}

\author{
Lucia De ANGelis ${ }^{1}$, Marco GONELLA ${ }^{1}$, Paola LetiZIA ${ }^{2}$, Paolo POLO ${ }^{2}$
}

1. MED Ingegneria S.r.1., Via Zangheri 16, 48100 Fornace Zarattini, Ravenne, Italie. lucia.deangelis@medingegneria.it; marco.gonella@medingegneria.it

2. MED Ingegneria S.r.1., Corso Stati Uniti 1/17, 35127 Padoue, Italie.

paola.letizia@medingegneria.it ; paolo.polo@medingegneria.it

\section{Résumé :}

La question de la protection du littoral relève d'une problématique de plus en plus d'actualité, notamment en rapport avec l'impact du changement climatique. En particulier, cette étude considère la région de Ravenne, où pendant les dernières années le développement incessant a conduit à la réalisation de beaucoup d'ouvrages et d'aménagements de protection. Ces ouvrages ont raidi un trait de cote naturellement très mobile et diminué la protection des sites urbanisés contre l'action de la mer. En même temps il était nécessaire de maintenir et de développer les principales activités de la région : le tourisme balnéaire et la capacité d'hébergement. Pour satisfaire cet objectif il faut garantir une extension convenable de la plage émergée et une bonne qualité de l'eau et des activités balnéaires. La présence de l'homme n'a pas seulement concerné les zones à terre, mais aussi les zones maritimes où l'on a construit beaucoup d'ouvrages rigides de défense côtière. Ceci a fortement altéré les conditions normales du transport sédimentaire et la morphodynamique côtière. En outre les zones côtières adjacentes sans ouvrages de défense sont déstabilisées par les effets des parties terminales des structures de défense. Le but de cette étude est d'identifier les interventions, souples et rigides, qui permettent de contrer, ou au moins de ralentir, le phénomène d'érosion le long de la côte, en essayant de créer un impact minimum sur l'environnement. On a suivi des méthodologies de défense côtière traditionnelles et innovatrices. Des solutions alternatives éco-compatibles respectant le milieu, et en particulier sa dynamique, sont nécessaires par rapport à celles qui ont existé jusqu'à nos jours, c'est-à-dire des solutions « dures » qui affectent l'équilibre du littoral. On doit donc recourir à des outils comme les modèles. Nous en présentons plusieurs types pour montrer la diversité des cas à traiter et, notamment, l'effort particulier que les scientifiques doivent déployer. Les analyses ont été faites avec l'utilisation de la modélisation numérique mono et bidimensionnelle, et en particulier grâce à l'utilisation du code de calcul MIKE21 et du logiciel LitPack développés par le Danish Hydraulic Institute. On a mené l'étude en collaboration avec les administrations publiques afin que les choix puissent être en accord avec les décisions politiques de gestion du territoire.

DOI: $10.5150 / \mathrm{cmcm} .2009 .046-6$ 


\section{Mots-clés :}

Gestion durable de la côte - Défense côtière - Modélisation numérique des phénomènes maritimes - Génie côtier - Innovation

\section{Introduction}

La zone étudiée est le littoral de Punta Marina, située au Sud du Port de Ravenne, en Italie. Pendant des années cette partie de la côte a vu la réalisation de beaucoup d'ouvrages rigides pour la défense côtière (brise-lames, épis submergés ou émergés) qui ont fortement altéré les conditions normales de transport sédimentaire et l'évolution naturelle du trait de côte. Les parties limitrophes aux zones intéressées par la construction de ces structures ont ressenti cette « rigidification » de la côte : l'apport de sédiments a beaucoup diminué et les effets des parties terminales des structures se sont révélés très importants. D'où la nécessité de contrer le phénomène érosif qui intéresse la zone non protégée au Nord du brise-lame submergé de Punta Marina, en évitant de provoquer d'autres dégâts de la partie côtière, maintenant stable, au Nord de l'épi "Ruvido".

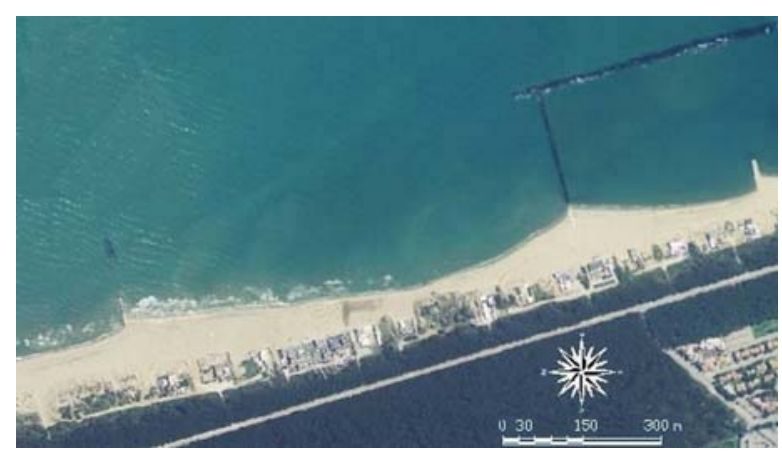

Figure 1. Zone étudiée, Punta Marina, Italie (Source: Google Earth).

\section{Méthodologie d'analyse}

L'analyse s'est déroulée en plusieurs phases, toutes effectuées de concert entre techniciens et organismes décisionnels :

1. Analyse de l'état actuel : levés bathymétriques et topographiques de la plage émergée et des dunes, levés des structures existantes, analyse sédimentologique, analyse de l'évolution du trait de côte, climat marin au large de Punta Marina à partir des données enregistrées par le système RON de Ancône (mesures de la houle) ;

2. Proposition de solutions alternatives, pour la diminution du phénomène érosif, soit "flexibles" (remblayage artificiel, culture de Posidonie), soit "rigides" (brise-lames, épis, optimisation des structures existantes), soit ouvrages non traditionnels (barre sous-marine et brise-lames submergés qui recréent les reefs);

3. Etude de 11 solutions possibles : 5 avec l'application du LitPack, qui utilise une schématisation de type monodimensionnelle des processus. Pour ce cas on a appliqué un climat marin moyen annuel. En particulier on a utilisé le modèle LitDrift (Littoral 
Drift) pour étudier la distribution et la quantité de dépôt sédimentaire engendré par les courants longitudinaux. En partant de ces résultats le module LitLine (Coastline profile) a été utilisé pour déterminer l'évolution du trait de côte. Enfin on a examiné l'évolution du profil bathymétrique transversal sous l'action d'une tempête de projet, en appliquant le modèle LitProf (Coastline profile). Les cinq autres solutions, dans lesquelles sont particulièrement évidents les phénomènes bidimensionnels, ont été analysées en appliquant les modèles bidimensionnels Parabolic Mild Slope waves (PMS), HydroDynamic (HD) et Sediment Transport (ST) du code de calcul MIKE21,. Le modèle PMS a été appliqué pour étudier les transformations subies par les houles ( 4 morphologiques et 4 extrêmes) pendant leur propagation du large vers la côte à cause des phénomènes énergétiques de "shoaling" (diminution de la profondeur), friction au fond, réfraction, diffraction et déferlement. Ensuite, l'application du modèle HD a permis le calcul des courants et de l'augmentation du niveau provoqués par la force des houles dans la bande entre la zone de déferlement et le trait de côte. Enfin avec l'utilisation du modèle ST on a calculé la capacité de transport sédimentaire en suivant le même calcul des modèles PMS et HD, suite à l'action des houles et des courants, calculée précédemment ;

4. Définition et étude détaillée de la solution optimale, en appliquant la modélisation numérique décrite ci-dessus (modèles de MIKE21 et LitPack). Cette solution est de type expérimental, avec peu d'impact sur l'environnement, et concerne la création d'un reef artificiel le long des premiers $350 \mathrm{~m}$ et la formation avec sédiments fins d'une barre sous-marine pour les $500 \mathrm{~m}$ suivants. Au début on a prévu aussi un remblayage artificiel pour permettre un avancement du trait de côte afin d'avoir une largeur de plage presque constante tout au long de la zone en question.
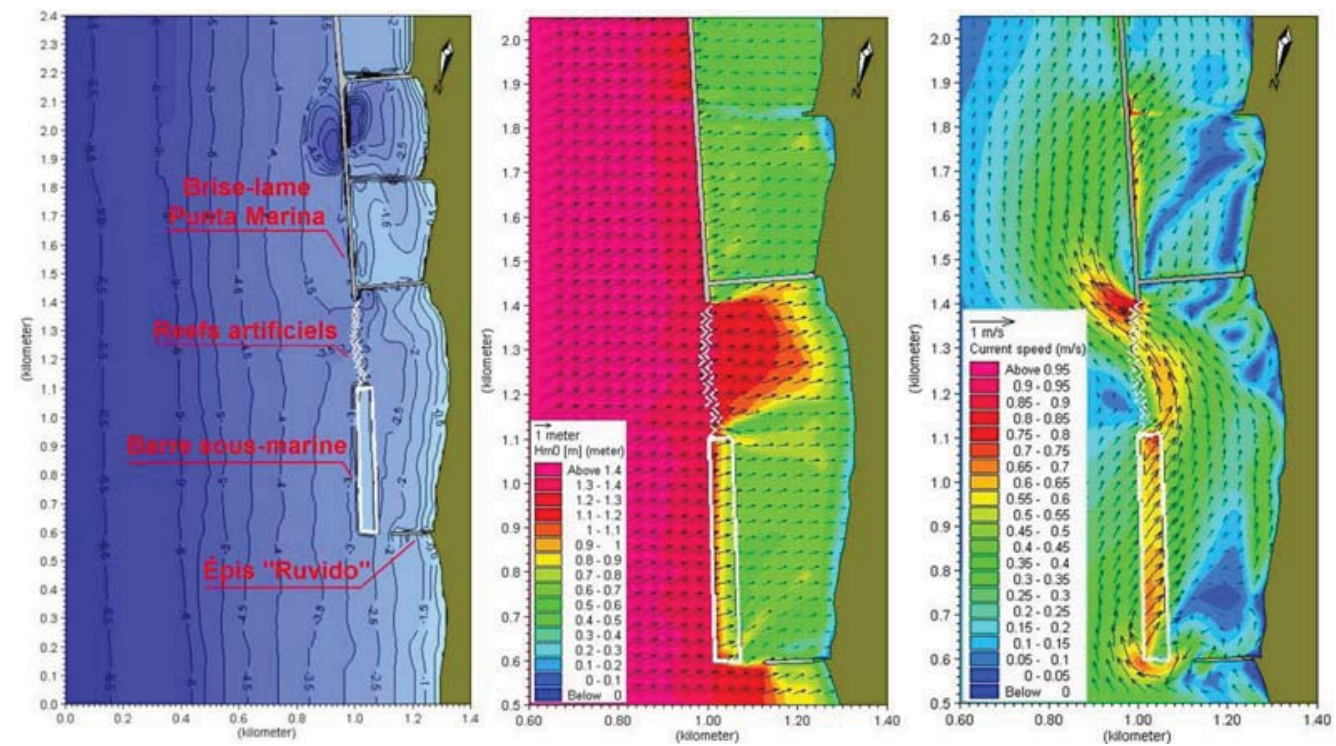

Figure 2. Bathymétrie de la zone, champs des hauteurs de houle et des vitesses calculés avec les modèles MIKE21-PMS et MIKE21-HD, configuration de projet. 


\section{Résultats obtenus}

1. L'insertion d'un épi intermédiaire n'apporte pas d'amélioration aux conditions d'érosion parce que le trait de côte a de toute façon la tendance à atteindre sa position actuelle ;

2. Le courbure ou l'allongement du brise-lame, l'élimination ou l'allongement de l'épi "rugueux" n'apportent pas d'amélioration comme au point 1 décrit ci-dessus ;

3. L'allongement du brise-lame submergé de Punta Marina jusqu'à la section finale de l'épi "rugueux" permettrait de maintenir fixe le trait de côte, mais provoquerait un recul de celui-ci au Nord de l'épi "rugueux", qui est presque stable à l'état actuel ;

4. Les créations d'un reef artificiel et d'une barre sous-marine ont été étudiées en appliquant la modélisation numérique, tout en connaissant la faible efficacité des modèles dans ces cas. En effet, pour les reefs artificiels, il est impossible de représenter la forme particulière et la composition en 3D des structures; pour la barre sous-marine il est impossible de représenter la variation de granulométrie du sable, l'incessante modification et l'interaction de la formation sablonneuse avec l'action des houles et des courants, vu que le modèle bidimensionnel employé est à fond fixe.

\section{Développements futurs}

La solution de projet choisie comme définitive représente, en Italie, une nouvelle typologie d'intervention pour la défense côtière et donc elle est complètement expérimentale. En considérant les nombreux facteurs qui interviennent dans l'application de cette méthodologie de défense, la seule façon d'étudier sa réussite est un monitoring directement sur le terrain. Cette stratégie de défense du littoral pourra donc représenter aussi une importante occasion pour les études et la recherche dans différents domaines : ingénierie, géologie, écologie, maritime, économique-social. Il faudra aussi créer des réunions d'information et de diffusion des connaissances obtenues pour optimiser le projet et les dimensions des structures des reefs artificiels et des barres sous-marines dans d'autres sites.

\section{Bibliographie}

DHI -Danish Hydraulic Institute- (2004). MIKE21, LIPACK Manuals, Horsholm, Denmark.

FONTOLAN G. (2003). Jumeira Beach - Umm Sequeim 2 Littoral Tract. Result of the monitoring surveys during September 2002 and August 2003. Università degli Studi di Trieste, Dipartimento di Scienze Geologiche Ambientali e Marine, Trieste, Italia.

GRUNNET N.M., RUESSINK B.G. (2004). Morphodynamic response of nearshore bars to a shoreface nourishment. Institute for Marine and Atmospheric Research, Utrecht, The Netherlands.

ISPRA (site web). http://www.idromare.it/

TECNOREEF (site web). http://www.tecnoreef.it/

US DEPARTMENT OF THE ARMY (1984). Shore Protection Manual. Coastal Engineering Research Center, Washington, USA.

VAN RIJN L.C., WALSTRA D.J.R. (2004). Analysis and modelling of shoreface nourishments. Delft Hydraulics, Delft, The Netherlands. 